\title{
Bespoke surgery: We're virtually there
}

\author{
Jonathan M. Chen, MD
}

\author{
From Congenital Cardiac Surgery, Seattle Children's Hospital, Seattle, Wash; and Pediatric Cardiovascular Sur- \\ gery, University of Washington School of Medicine, Seattle, Wash. \\ Disclosures: Author has nothing to disclose with regard to commercial support. \\ Received for publication Dec 15, 2017; accepted for publication Dec 27, 2017; available ahead of print Feb 2 , \\ 2018. \\ Address for reprints: Jonathan M. Chen, MD, University of Washington School of Medicine, 4800 Sand Point \\ Way NE - RC 2.820, Seattle, WA 98105 (E-mail: jmchen@uw.edu). \\ J Thorac Cardiovasc Surg 2018;155:1743-4 \\ $0022-5223 / \$ 36.00$ \\ Copyright (C) 2018 by The American Association for Thoracic Surgery \\ https://doi.org/10.1016/j.jtcvs.2017.12.109
}

An image often used as the introductory slide for any lecture on the Fontan procedure displays a dizzying array of various versions of the operation in its evolution since inception. ${ }^{1}$ From initial atriopulmonary connection to "leaner and meaner" prosthetic tube graft, all seek to optimize passive pulmonary blood flow. Indeed, any perturbations in the connection can lead to distorted flow distribution, poor pulmonary arterial growth, stasis/ thrombosis, or power loss and resultant reduction in cardiac output in this classically unforgiving physiology. Accordingly, recent strategies have sought to use flow dynamics in an effort to guide preoperative planning and customize the Fontan pathway for individual patients. ${ }^{2,3}$ However, these tactics have been partially limited by the need to select prosthetic grafts "off the rack" - the dream of a tailor-made conduit, optimized for specific anatomy and flow efficiency, has remained elusive until now.

In this issue of the Journal, Siallagan and colleagues ${ }^{4}$ describe an important first step toward this goal. Their analysis first pitted cardiac magnetic resonance imaging data from 2 patients after Fontan completion against idealized virtual models (both tube and bifurcated conduits) and later used models in a 4-chamber flow loop, as well for the basis of 3-dimensional tissue-engineered vascular grafts (with the anticipation of in vivo applications in the future). On the basis of these data, one of their patients would theoretically enjoy the benefit of a bifurcated graft-with very specific distal anastomotic sites for each limb-whereas the other saw little advantage over a simple (albeit customized) tube graft. Indeed, the term "advantage" here must be used somewhat cautiously, because it is only speculation that the creation of more balanced hepatic flow distribution portends a better outcome; in a more fundamental way, "power loss" in this investigation was used as a surrogate of cardiac output, where obviously in the human condition it is only one of its many determinants. Additionally, the fundamental assumptions of a rigid Fontan

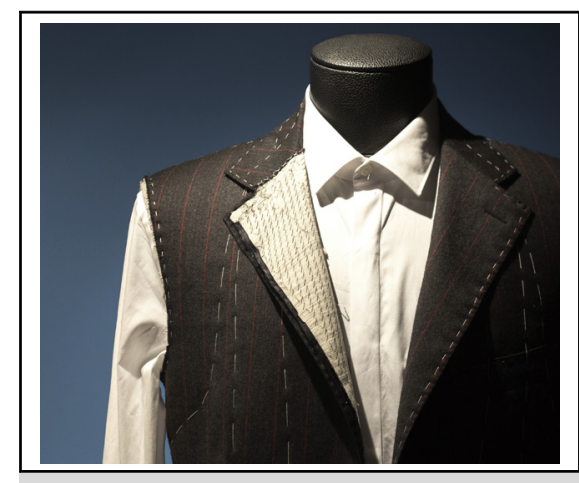

Off the rack or custom-made?

Central Message

Customized conduits could be a great advance for the Fontan procedure. With the use of magnetic resonance imaging data, ideal grafts can be created for optimum efficiency; the clinical impact of these constructs is yet unknown.

See Article page 1734 .

geometry and of laminar flow (ie, not during exercise or across full cardiac cycles) disclose obvious next areas of focus for "version 2.0".

Without question, the proof will be in the Fontan pudding. Thrombogenicity has always been a central concern with both tissue-engineered vascular graft conduits and bifurcated polytetrafluoroethylene (PTFE) grafts - the former because of the material, the latter because of each limb's limited cross-sectional area. Whether patients (or animals) actually derive benefit from a more balanced and efficient custom-made circuit is still unclear. What is irrefutable, however, is that the Fontan procedure is an imperfect construct applied to a broadly heterogeneous patient population and undoubtedly could be perfected. The "one size fits all" approach that we as a specialty have applied for decades surely needs to enter the era of the bespoke; to this end, the fashionista battle cry may have no better application than in Fontan physiology, because let's face it, "if you look good, you feel good."

\section{References}

1. Fontan F, Baudet E. Surgical repair of tricuspid atresia. Thorax. 1971;26: 240-8.

2. Kanter KR, Haggerty CM, Restrepo M, deZelicourt DA, Rossignac J, Parks WJ et al. Preliminary clinical experience with a bifurcated Y-graft Fontan procedure-a feasibility study. J Thorac Cardiovasc Surg. 2012;144:383-9. 
3. Haggerty CM, deZelicourt DA, Restrepo M, Rossignac J, Spray TL, Kanter KR, et al. Comparing pre- and post-operative Fontan hemodynamic simulations: implications for the reliability of surgical planning. Ann Biomed Eng. 2012;40:2639-51.
4. Siallagan D, Loke Y, Oliveri L, Opfermann J, Ong CS, deZelicourt D, et al. Virtual surgical planning, flow simulation, and 3-dimensional electrospinning of patientspecific grafts to optimize Fontan hemodynamics. J Thorac Cardiovasc Surg. 2018;155:1734-42. 